\title{
Control System Design for a Centrifuge Motion Simulator Based on a Dynamic Model
}

\author{
Jelena Vidaković1,* - Vladimir Kvrgić2 ${ }^{1}$ - Mihailo Lazarević3 \\ 1 University of Belgrade, Lola Institute, Serbia \\ 2 University of Belgrade, Institute Mihajlo Pupin, Serbia \\ 3 University of Belgrade, Faculty of Mechanical Engineering, Serbia
}

This paper presents a dynamic model-based design of a control system and an approach toward a drive selection of a centrifuge motion simulator (CMS). The objective of the presented method is to achieve the desired performance while taking into account the complexity of the control system and the overall device cost. An estimation of a dynamic interaction of the interconnected CMS links motions is performed using the suitable inverse dynamics simulation. An algorithm based on the approximate inverse dynamics model is used within the drive selection method. The model of the actuator's mechanical subsystem includes the effective inertia (inertia reflected on the rotor shaft) calculated from the inverse dynamics model. A centralized control strategy based on a computed torque method is considered and compared to traditional decentralized motion controllers. To obtain an accurate comparison of the suggested control methods through a realistic simulation, structural natural frequencies of the manipulator links are considered, and the actuator capabilities are taken into account. The control system design and simulation methods and the drive selection strategies, presented here for the CMS, are applicable within the general robot manipulator's domain.

Keywords: robot dynamics, robot control, computed torque method, centrifuge

Highlights

- A dynamic model-based control system design of a centrifuge motion simulator.

- A dynamic model-based approach toward an adequate drive selection of a centrifuge motion simulator.

- Realistic simulation of the control system takes into account natural frequencies of the mechanical structure, actuator torque limits and inertia reflected on the rotor shafts.

\section{INTRODUCTION}

Pilots of the newest generation of combat aircraft are exposed to dangerous effects of high $G$ forces and sudden angular movements in all three aircraft axes, with accelerations of up to $9 g\left(g=9.81 \mathrm{~m} / \mathrm{s}^{2}\right.$ is Earth's gravitational acceleration), and acceleration rates of up to $9 \mathrm{~g} / \mathrm{s}$. Herein, the acceleration force $G=a / g$ corresponds to the magnitude of acceleration acting on the pilot, $a=\left(a_{n}^{2}+a_{t}^{2}+g^{2}\right)^{1 / 2}$, where $a_{n}$ and $a_{t}$ are the normal and tangential accelerations, respectively [1]. Under such conditions, the pilot's ability to control the aircraft is reduced, and the pilot may suffer from a loss of consciousness induced by high $G$ loads (G-LOC). Researchers are seeking answers to whether pilots can cope with the physiological and psychological requirements placed upon them with the newest generation of fighter aircraft [2]. High $G$ training in a centrifuge motion simulator (CMS) is used to artificially increase the inertial force under controlled conditions. In this study, 'open loop flight simulations', with predefined trajectories, are considered.

The virtual structure of a CMS is made using CATIA, as shown in Fig. 1. The CMS is modelled and controlled as a 3 degrees of freedom (DOF) robot manipulator with revolute joints. The pilot seat (or chest/head) acts as an end-effector. In Fig. 2, rotational axes and frames of the CMS are given. The CMS arm rotates about the vertical axis. It carries a ring that rotates about the roll axis, and a gondola rotating about the pitch axis. Denavit-Hartenberg notation is used for geometry model development [3]. Frame with index 3 is attached to the end-effector (the pilot's seat). An inverse dynamics (ID) algorithm, which computes the torques to be applied to the joints in order to obtain their commanded motions, for the CMS is given in [1] in the form of recursive Newton-Euler equations.

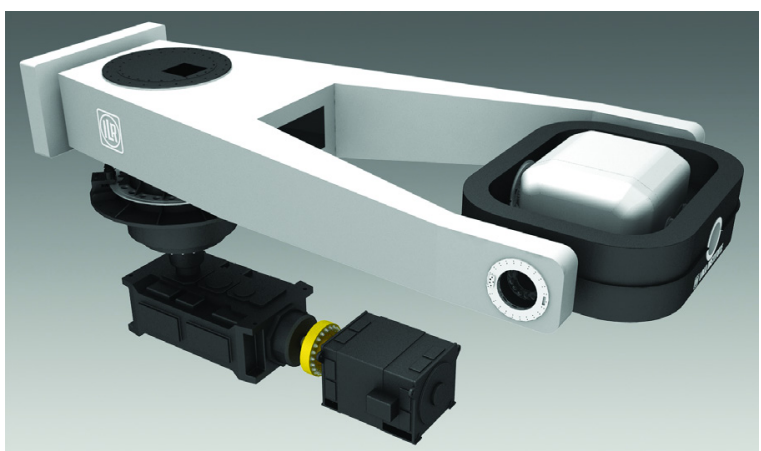

Fig. 1. CMS with 3 DOF 


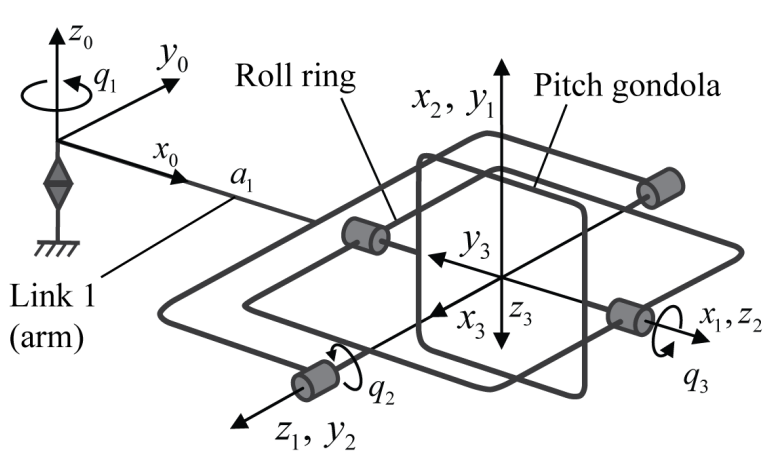

Fig. 2. CMS rotational axes and frames

Robot control is a challenging task due to high nonlinearity of robot dynamics and coupling effects between the joints. To deal with the nonlinearity and time variability of robot dynamics, many advanced control strategies have emerged, including adaptive control [4] and [5], sliding mode control [6], intelligent control [7] and [8], etc. Regarding the problem of uncertainty of kinematic and dynamic robot parameters, control schemes that make use of soft computing methods including neural networks [9] to [11] and fuzzy logic [12] have been developed. In order to improve trajectory tracking, various optimization techniques are used within motion controllers, [13] to [15]. Joint space control methods, which are adequate in situations in which the manipulator tasks can be accurately preplanned and little or no online trajectory adjustments are necessary [16], can be classified into decentralized (when a single manipulator joint is controlled independently of the others) and centralized (when dynamic interaction effects among the joints are considered) techniques [17]. The advantage of one particular control method over another depends on the robot application, performance requirements, characteristics of the mechanical design and actuators, implementation requirements, etc. A CMS falls into the category of high-assurance systems requiring a high level of robustness in terms of system performance and a guaranteed set of critical properties including reliability, availability, safety, and security [18]. The application of empirically validated control methods in systems in which their failure can result in a loss of life or property is questionable and must always be backed up by techniques that minimize the possibility of such undesirable effects.

This paper deals with a control system design and a drive system selection of the CMS that takes into account the desired performance, the control system complexity, and the overall system cost.

There are very few papers in the literature that deal with CMS motion control. In [19], a similar centrifuge, driven with hydraulic actuator system, is presented. The centrifuge arm is controlled with conventional proportional-derivative (PD) speed feedback, while fuzzy sliding mode control is applied for the roll and pitch angles control. Simulation results for the trajectory tracking are given, divided into the increasing and decreasing $G$ load profile segments without transitions in $G$ load rates. In [20], another realization of the centrifuge is described, with the study of control methods based on the concepts of adaptive feedback linearization; some numerical simulation is provided for the less challenging trajectories.

Within a choice of the prospective control system for a robot manipulator in practice, complexity and implementation requirements may play significant roles. In regard to the potential use of advanced control schemes, given that such utilization can lead to complex control structures, benefits have to be clearly determined in comparison to the simpler control strategies. In this perspective, within the control system design for the CMS questions arise regarding whether a dynamic model should be taken into account, and if so, how. Methods used to answer this question for the CMS are presented herein.

In this study, the thorough analysis and suitable simulation of the CMS manipulator's dynamic model are used for an assessment of the nature of the mutual influences of the interconnected links' motions, from which conclusions on the correct control strategy regarding both the performance and the complexity are drawn. On the basis of the results of the presented ID model-based simulations, a single joint servo control is proposed for the CMS arm control. For the second (ring) and third (gondola) axes, the addition of a feedforward computed torque compensator [21] to the feedback controller is considered.

To obtain an accurate comparison of the suggested control methods through a realistic simulation, the following conditions are met: 1) the effective inertia (inertia reflected on the rotor shaft), which provides a realistic process model, is calculated from the ID for the desired joint trajectories and chosen actuator model, 2) the choice of feedback controller gains considers the structural natural frequencies (herein obtained using CATIA software), and 3) the torque limits for the chosen actuators are included in the simulation of the controlled process. Compared to the previously published work in [19] and [20], the performance of the considered control methods is here given for the significantly more challenging CMS trajectory profile, with the maximum acceleration magnitude of $9 g$, the maximum acceleration rate of 
$\pm 9 g$ per second, and with provided transitions in $G$ load rates.

A CMS falls into the category of heavy machinery (herein, the CMS arm length $a_{\mathrm{r}}$ is $8 \mathrm{~m}$, its mass is approximately 42 tons, the whole assembly mass is 45 tons). Combined with the challenging joint trajectories, actuators with desired powers have huge weights. It is essential for motors to have sufficient power to rotate the links rapidly as well as to be lightweight. Bigger actuators induce an increase in manipulator inertia, energy consumption, and cost of the overall system.

In this study, a procedure based on the approximate inverse dynamics (aID) model [1], is used within the drive selection problem. The presented procedure enables the choice of actuators in terms of their power and weights in such a way that the smallest actuators with the potential to achieve the required joints' motions are determined. When the actuators are chosen, their model parameters are included within control system simulations.

The remainder of this paper is organized as follows. In section 1, the methodology for the design of the motion control system and the selection of CMS drives based on dynamic manipulator modelling are presented. Results and a discussion are provided in section 2. Concluding remarks are given in section 3.

\section{DYNAMIC MODEL-BASED DESIGN OF CMS MOTION CONTROLLER}

The required task for the CMS manipulator is to achieve commanded acceleration force $G$ at the centre of the gondola, where the pilot's seat, chest, or head is accommodated. The desired tasks are defined using an application program written in L-IRL robot programming language, modified for flight simulators [22]. A trajectory planner for the CMS is implemented in L-IRL through the algorithm used to calculate the joint trajectories $q_{k}, \dot{q}_{k}, \ddot{q}_{k}, k=1,2,3$ which produce the desired change in $G$-force at the centre of the gondola with specified jerks of $G,[1]$ and [23]. For the commanded end-effector acceleration ( $G$ load) given in Fig. 6, the positions, velocities, and accelerations of the CMS joints, $q_{k}, \dot{q}_{k}, \ddot{q}_{k}, k=1,2,3$ obtained as the outputs of the trajectory planner, are given in Fig. 3. Challenging kinematic profiles with the rapid changes in velocity and large jump-like changes in acceleration, particularly for axes 2 and 3 , are required.

On the basis of the maximum values of the actuating torques obtained from the ID model for the most demanding commanded CMS trajectory, the initial selection of the corresponding actuators is
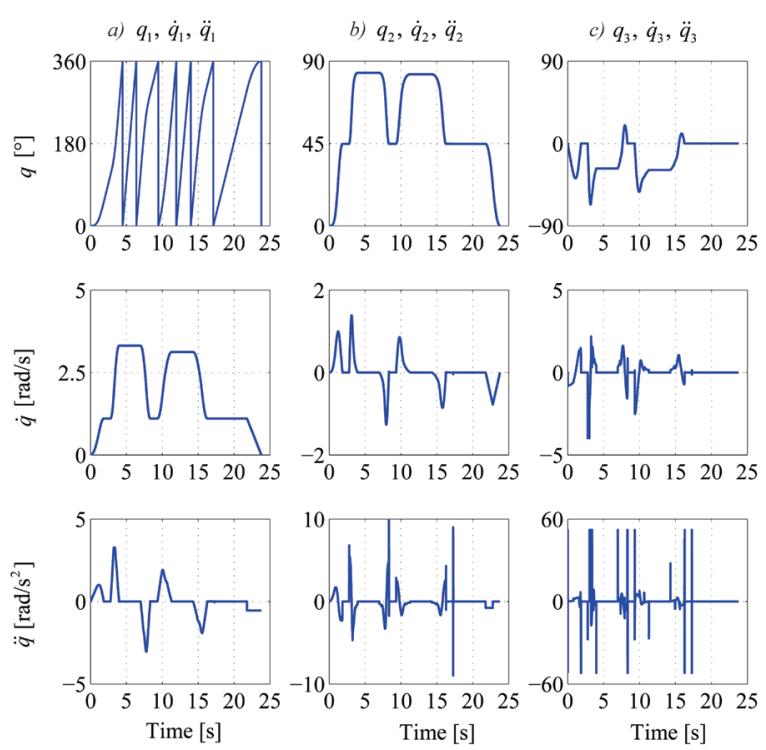

Fig. 3. Positions, velocities, and accelerations of CMS joints; a) $k=1, b) k=2$, and c) $k=3$

performed [1]. For the first axis (centrifuge arm), a DC motor with a rated torque of $M_{1 \mathrm{r}}=41200 \mathrm{Nm}$ was chosen. The motor overload capability is 2 ; the chosen gearbox has a gear ratio of 16.5 and an efficiency of $\eta_{1}=0.94$. This gives a short time maximum torque of $M_{1 \max }=1.28 \cdot 10^{6} \mathrm{Nm}$ (for less than $5 \mathrm{~s}$ ). For the axes 2 and 3, the actuating system consisting of two torque motors with a maximum torque of $M_{i \max }=10900 \mathrm{Nm}$ [24] is considered for each axis.

Motion equation for a manipulator with $n$ degrees of freedom can be described in the following matrix form:

$$
\mathbf{D}(\mathbf{q}) \ddot{\mathbf{q}}+\mathbf{H}(\mathbf{q}, \dot{\mathbf{q}}) \dot{\mathbf{q}}+\mathbf{g}(\mathbf{q})=\tau,
$$

where $\mathbf{D}(\mathbf{q})$ is $n \times n$ inertia/mass matrix, $\mathbf{H}(\mathbf{q}, \dot{\mathbf{q}})$ is an $n \times n$ matrix of centrifugal and Coriolis terms, $\mathbf{g}(\mathbf{q})$ is $n \times 1$ is a vector of gravitational terms and $\boldsymbol{\tau}$ is $n \times 1$ vector of the actuating torques. For an easier description regarding control methods used herein for the CMS, Eq. (1) is rewritten in Eq. (2), $k=1,2, \ldots, n$, as a set of $n$ coupled nonlinear differential equations.

$$
\sum_{j=1}^{n} d_{k j}(\mathbf{q}) \ddot{q}_{j}+\sum_{j=1}^{n} \sum_{i=1}^{n} h_{k j i}(\mathbf{q}) \dot{q}_{j} \dot{q}_{i}+g_{k}(\mathbf{q})=\tau_{k} .
$$

With the recursive Newton-Euler method, the actuating torque $\tau_{k}$ in Eq. (2) is obtained as the $z$ component of the vector $\widehat{\mathbf{m}}_{k}$, which is a moment exerted on link $k$ by link $k-1$, described in the frame attached to joint $k-1$ [25], i.e. $\tau_{k}=\widehat{m}_{z k}$. Hereafter, an over-line hat notation $\left({ }^{\wedge}\right)$ along with subscript $k$ is used to denote the matrices, vectors, and their 
components described in the frame attached to link $k$. From Eqs. (1) and (2), it is clear that when large operational speeds and accelerations are required, the nonlinear coupling terms may have a strong influence on the system performance. In this case, it is advisable to design a control system that takes advantage of the detailed knowledge of manipulator dynamics [17].

\subsection{Determination of Dynamics Effects of the CMS Interconnected Links' Motions}

In this paper, the mutual dynamic effects of the interconnected links' motions of the CMS are analysed using ID-based simulations. For a specific $k^{\text {th }}$ joint, influences of the other links motions, $j=1,2, \ldots, n$, $j \neq k$, are estimated through their contribution within the value of the $k^{\text {th }}$ joint actuating torque. Following ID simulations are used for this purpose.

For a specific commanded motion of the manipulator, outputs of the trajectory planner are obtained in the form of the time sequences of the joints' trajectories $\tilde{q}_{j}, \dot{\tilde{q}}_{j}, \ddot{\tilde{q}}_{j}, j=1,2, \ldots, n$, which are used as the input for the ID algorithm, Eq. (2), in order to obtain the time sequences of the corresponding actuating torques realizing the given manipulator motion, $\tilde{\tau}_{j}, j=1,2, \ldots, n$. Next, for a specific $k^{\text {th }}$ joint, $k=1,2, \ldots, n$, ID algorithm, Eq. (2), is used to calculate the time sequence of actuating torque $\tilde{\tau}_{k Z}$ for the same trajectory of that joint $q_{k Z}, \dot{q}_{k Z}, \ddot{q}_{k Z}=\tilde{q}_{k}, \dot{\tilde{q}}_{k}, \ddot{\tilde{q}}_{k}$, but with the trajectories of the other joints that are equal to zero, $q_{j z}, \dot{q}_{j Z}, \ddot{q}_{j Z}=0, j=1,2, \ldots, n, j \neq k$ i.e. as if there are no rotations of the other links about their corresponding axes.

If the comparison of the obtained torques $\tilde{\tau}_{k}$ and $\tilde{\tau}_{k Z}$ gives insignificant differences for the most demanding CMS motions, it can be concluded that motions of the other joints do not significantly contribute the required actuating torque for the joint $k$. Consequently, the effects of the nonlinear coupling terms in Eq. (2) for that particular joint may be reasonably treated as disturbances that can be easily overcome using a single joint feedback controller.

\subsection{Usage of alD Algorithm within a Selection of the CMS Drives}

When high accelerations are required within a manipulator system, minimizing the system inertia is necessary to satisfy the dynamic performance [26]. For this reason, the actuators of the CMS are herein chosen to operate very close to the maximum motor torque. Here, it is necessary to check whether the desired trajectories can be achieved, i.e., if the required joint torques are less than or equal to the maximum motor torques. If the desired actuating torques exceed the maximum level for a very small value, a trajectory modification using an algorithm based on aID described in [1] is applied.

In aID, a discretization method in which the desired joint velocities $\dot{q}_{k}$ for a specific interpolation period are calculated as functions of the programmed accelerations for that interpolation period as $\dot{q}_{k}=\dot{q}_{k \text { prev }}+\ddot{q}_{k} \Delta t, \quad k=1,2, \ldots, n, \quad$ is used within calculations of the actuating joint torques $\tau_{k}=\hat{m}_{z k}$, Eq. (2). Hereinafter, the suffix "prev" indicates a value in the previous interpolation period. Subsequently, the following will be valid for the products $\dot{q}_{j} \dot{q}_{i}$ within $h_{k j i}(\mathbf{q}) \dot{q}_{j} \dot{q}_{i}$ terms in Eq. (2):

$$
\dot{q}_{j} \dot{q}_{i}=c_{j i}+a_{i} \ddot{q}_{j}+a_{j} \ddot{q}_{i}+\ddot{q}_{j} \ddot{q}_{i} \Delta t^{2},
$$

with $c_{j i}=\dot{q}_{\text {jprev }} \dot{q}_{\text {iprev }}, a_{i}=\dot{q}_{i \text { prev }} \Delta t, a_{j}=\dot{q}_{\text {jprev }} \Delta t$. Given that the interpolation period of $\Delta t=5 \mathrm{~ms}$ is used within the control unit, if the terms with $\Delta t^{2}$ are neglected in Eq. (3), the error of $\ddot{q}_{j} \ddot{q}_{i} \Delta t^{2}=2 \cdot 10^{-5} \ddot{q}_{j} \ddot{q}_{i}$ is made. In the consecutive section, it will be described how the motions of the links 2 and 3 have an insignificant effect on the motion of the CMS arm (link 1). For this reason, the CMS arm is excluded from the following calculations. After neglecting $\Delta t^{2}$, joint torques $\tau_{2}=\widehat{m}_{z 2}$ and $\tau_{3}=\widehat{m}_{z 3}$ are obtained from Eq. (2) for a specific interpolation period in the following approximate form:

$$
\begin{aligned}
& \tau_{2}=c_{2}+c_{22} \ddot{q}_{2}+c_{23} \ddot{q}_{3}, \\
& \tau_{3}=c_{3}+c_{32} \ddot{q}_{2}+c_{33} \ddot{q}_{3} .
\end{aligned}
$$

Simulations for various CMS trajectories have shown that for $\Delta t=5 \mathrm{~ms}$, joint torques $\tau_{2}$ and $\tau_{3}$ given in Eqs. (4) and (5) differ by less than $1 \%$ from the results obtained by a classical ID (without neglecting the terms with $\Delta t^{2}$ in Eq. (2)). Coefficients $c_{2}, c_{22}, c_{23}$, $c_{3}, c_{32}, c_{33}$ in Eqs. (4) and (5) are calculated for every interpolation period as functions of variables $\ddot{q}_{1}, \dot{q}_{2 \text { prev }}, \dot{q}_{3 \text { prev }}, q_{1}, q_{2}, q_{3}$ and constants $\Delta t$, the centrifuge arm length $a_{\mathrm{r}}$, components of the inertia matrices $\hat{\mathbf{I}}_{2-4}$ of links $k=2,3,4$ about the centre of mass of link $k$ (here, index 4 indicates an external load, i.e., the pilot and the pilot's seat and equipment), components of $\hat{\mathbf{r}}_{2-4}$ representing position vectors of the centre of mass of links 2 and 3 and an external load with respect to the origin of the frame attached to link $k$, and the masses of links 2 and 3 , and the mass of an external load.

Next, the check and, if necessary, the limitation of the joint torques to the maximum values $\tau_{2 \max }$ and $\tau_{3 \max }$ achievable by the motors is performed in the 
following manner: If it is determined that, for the given interpolation period, joint torque/s $\tau_{2}$ and $\tau_{3}$ exceed the maximum value/s, the maximum possible values of accelerations of $\ddot{q}_{2}$ and $\ddot{q}_{3}$ that the motors can achieve in this interpolation period are calculated by solving the system described in Eqs. (4) and (5) for the maximum values of the moments $\tau_{2}$ and $\tau_{3}$ :

$$
\begin{aligned}
& \ddot{q}_{2 \max }=\frac{\left(\tau_{2 \max }-c_{2}\right) c_{33}-\left(\tau_{3 \max }-c_{3}\right) c_{23}}{c_{22} c_{33}-c_{23} c_{32}}, \\
& \ddot{q}_{3 \max }=\frac{\left(\tau_{3 \max }-c_{3}\right) c_{22}-\left(\tau_{2 \max }-c_{2}\right) c_{32}}{c_{22} c_{33}-c_{23} c_{32}} .
\end{aligned}
$$

New angular velocities and joint positions are then obtained from Eqs. (6) and (7) using a numerical integration. When this procedure is implemented, check if the modification of the end-effector trajectory is within the allowable limits needs to be performed. If it is, the same procedure can be used for the next lower power motor, such that the smallest actuator that meets the specified requirements is found. After completion of this procedure, the aID algorithm is regularly used within the trajectory planner.

The major benefit of the aID implementation is that modification of the trajectories of a specific joint are not only due to that actuator saturation, but also to the saturation of the other joints' actuators, such that they facilitate the other links' motions when the other actuators are saturated. The appropriate selection of actuators will ensure that modifications of the joint trajectories produce negligible changes in the obtained $G$ load.

\subsection{Design of the CMS Motion Controllers}

In this study, a traditional decentralized single joint feedback control is compared with the dynamic model-based strategy for the CMS. To obtain realistic simulations that can provide relevant insight, a credible process model based on the effective inertia calculated from the ID is used. With structural natural frequencies and actuator capabilities taken into account, a realistic simulation of the controlled process is achieved.

\subsubsection{Model of Actuator's Mechanical Subsystem}

The equation of motion for rigid body rotation about an axis is given in Eq. (8), [27]:

$$
I \ddot{q}=\tau_{\mathrm{M}}-\tau_{\mathrm{L}}-B \dot{q},
$$

where $q$ is a rotational position, $I$ is the inertia of the moving parts, $B$ is equivalent system friction coefficient, $\tau_{\mathrm{M}}$ represents the driving torque, and $\tau_{\mathrm{L}}$ is the load torque. If Eq. (8) is applied to the mechanical subsystem of a robot joint actuator, $I$ depend on the instantaneous manipulator configuration, $B$ is the nonlinear function of the rotor speed. Load torque $\tau_{\mathrm{L}}=\tau_{\mathrm{LD}}+\tau_{\mathrm{LS}}$ is of a twofold nature: predictable part $\tau_{\mathrm{LD}}$ caused by the motion of the chain of interconnected links which can be calculated from the manipulator dynamic model, and torque due to stochastic disturbances $\tau_{\mathrm{LS}}$. Herein, it is assumed that there are no other predictable disturbances contributing to motor load torque. Following the nonlinear time-variant model of joint $k$ actuator's mechanical subsystem is obtained:

$$
I_{\mathrm{effk}} \ddot{q}_{\mathrm{m} k}+B_{\mathrm{efft} k} \dot{q}_{\mathrm{m} k}=\tau_{\mathrm{M} k}-\tau_{\mathrm{L} k} .
$$

Here, $\dot{q}_{\mathrm{m} k}=r_{k} \dot{q}_{k}$ is the angular speed of the actuator's rotor; $r_{k}$ is the motor gear ratio; $B_{\text {effk }}$ is the equivalent (reflected) friction coefficient; $I_{\text {eff } k}$ is the inertia reflected onto the rotor shaft, denoted here as the effective inertia; and $\tau_{\mathrm{M} k}$ is the driving torque generated by the actuator of the $k^{\text {th }}$ joint. Herein, the load torque $\tau_{\mathrm{LD} k}$ is calculated from ID model, Eq. (2), in the following manner [28]:

$$
\begin{aligned}
\tau_{\mathrm{LD} k}= & \frac{\tau_{k}-d_{k k} \ddot{q}_{k}}{r_{k}}= \\
& \frac{\sum_{j=1, j \neq k}^{n} d_{k j}(\mathbf{q}) \ddot{q}_{j}+\sum_{j=1}^{n} \sum_{i=1}^{n} h_{k j i}(\mathbf{q}) \dot{q}_{j} \dot{q}_{i}+g_{k}(\mathbf{q})}{r_{k}},
\end{aligned}
$$

that is, joint torque $\tau_{k}=\hat{m}_{z k}$ from Eq. (2) is reduced for the inertial term $d_{k k} \ddot{q}_{k}$. Adding a coefficient of $\ddot{q}_{k}$ in ID, Eq. (2), to motor and gearbox inertia $I_{\mathrm{m} k}$, the effective inertia for the joint $k$ actuator is obtained as [28]:

$$
I_{\text {effk }}=\left(I_{\mathrm{m} k}+d_{k k} / r_{k}^{2}\right) \text {. }
$$

If an accurate model for the friction experimentally obtained is available, the friction compensation can be introduced into the control. An alternative approach is to regard the bounded nonlinear friction terms as disturbances in traditional servo systems [29]. For the torque motors, chosen for the actuation of axes 2 and 3, the friction problem is of much less significance compared to motors with a gear reduction and can be regarded as negligible. With this approach, Eq. (9) becomes:

$$
I_{\mathrm{effk}} \ddot{q}_{\mathrm{m} k}=\tau_{\mathrm{M} k}-\tau_{\mathrm{L} k} .
$$




\subsubsection{Computed Torque Method}

The computed torque method implies a feedforward cancelation of the nonlinear coupling terms in Eq. (2). Here, the load torque from the motion of the chain of interconnected links, $\tau_{\mathrm{LD} k}$, obtained from ID, Eq. (10), is cancelled with a feedforward signal. The addition of feedback is beneficial for improving the referencetracking capability of the control system given that modelling in practice is almost never error-free, and for suppressing the effects of stochastic disturbances. In Fig. 4, a block diagram of a single-input-singleoutput (SISO) system of the computed torque method with an added feedback controller is shown. After implementing the feedforward disturbance cancellation, the process model given in Eq. (12) becomes:

$$
I_{\text {effk }} \ddot{q}_{\mathrm{m} k}=u_{k}-\tau_{\mathrm{LSk}},
$$

where $u_{k}$ is the torque proportional to the feedback controller output.

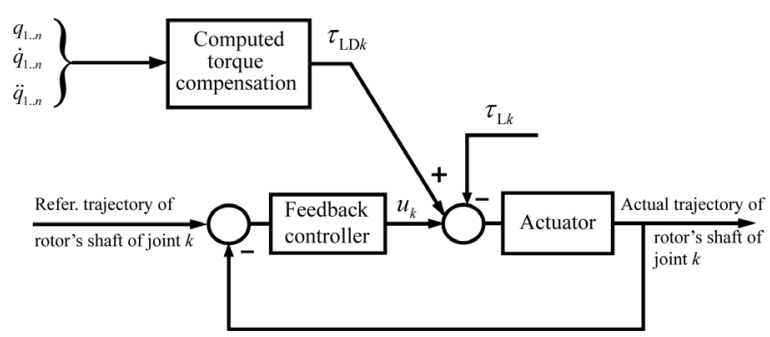

Fig. 4. Computed torque compensator with a feedback controller

\subsubsection{Determination of Feedback Controller Gains for the Simulation Model}

Given that the structural flexibilities in the system are not accounted for in the process modelling, to avoid exciting the unmodeled resonances, a rule of thumb is used when the lowest structural natural frequency $\omega_{\mathrm{r} k}$ of link $k$ is at least two times bigger than the natural (undamped) frequency $\omega_{\mathrm{n} k}$ of the feedback controller for joint $k$ [25]. To compare these frequencies, the PD position and proportional-integral (PI) velocity controllers are chosen, so that second-order closedloop system with a characteristic polynomial $s^{2}+2 \zeta_{k} \omega_{\mathrm{n} k} s+\omega_{\mathrm{n} k}^{2}$ (where $\zeta_{k}$ is the damping ratio) is obtained. Oscillations in the joint position or speed should be avoided.

Characteristic polynomials of the closed-loop system, where the rotor position $q_{\mathrm{m} k}$ and speed $\dot{q}_{\mathrm{m} k}$ are controlled using the PD and PI feedback, respectively, are given in the following forms:

$$
\begin{aligned}
& s^{2}+K_{\mathrm{DPk} k} s / I_{\text {effk }}+K_{\mathrm{PP} k} / I_{\mathrm{effk} k}, \\
& s^{2}+K_{\mathrm{PS} k} s / I_{\mathrm{effk}}+K_{\mathrm{ISk} k} / I_{\mathrm{effk} k},
\end{aligned}
$$

where $K_{\mathrm{PP}}, K_{\mathrm{DP}}, K_{\mathrm{PS}}, K_{\mathrm{IS}}$ are the proportional and derivative, and proportional and integral, gains of the PD position and PI speed feedback, respectively.

A simple solution to overcome the problem of variable process control is to adopt a time-invariant model, tune the controller for the largest load, and accept the deteriorated performance under other operating conditions [30]. For the PD position control, Eq. (14), the effective inertia of a specific $k^{\text {th }}$ joint actuator, Eq. (11), depends on the instantaneous manipulator configuration. For a fixed proportional gain $K_{\mathrm{PP} k}$, the natural frequency of the closed-loop system in Eq. (14) varies, i.e., $\omega_{\mathrm{n} k}(\mathbf{q})=\sqrt{K_{\mathrm{PPk}} / I_{\text {effk }}(\mathbf{q})}$. The maximum value of the natural frequency is obtained for the minimum value of the effective inertia. However, the damping ratio $\zeta_{k}=K_{\mathrm{DP} k} /\left(2 I_{\text {effk }}(\mathbf{q}) \omega_{\mathrm{n} k}(\mathbf{q})\right)=K_{\mathrm{DP} k} /\left(2 \sqrt{I_{\text {effk }}(\mathbf{q}) K_{\mathrm{PP} k}}\right)$ has the smallest value for the maximum value of the effective inertia $I_{\text {effkmax }}$, Eq. (11). Considering the request in which the motion of the link is never underdamped, if the value of $\zeta_{k}$ for $I_{\text {effkmax }}$ is 1 (critically damped response), in all configurations with smaller values of the effective inertia motions will be overdamped. In this way, fastest response without oscillations is obtained. For $I_{\text {effk }}=I_{\text {effkmax }}$, the relation between the proportional gain $K_{\mathrm{PP} k}$, Eq. (14), and natural frequency $\omega_{\mathrm{n} k}$ is as follows:

$$
K_{\mathrm{PPk}}=I_{\text {effkmax }} \omega_{\mathrm{nk}}^{2} \text {. }
$$

The maximum value of the allowed natural frequency $\omega_{\mathrm{n} \max k}=\omega_{\mathrm{r} k} / 2$ is used for obtaining the maximum proportional gain in Eq. (16), $K_{\mathrm{PP} \text { max }}=I_{\text {effl max }} \omega_{\mathrm{rk}}^{2} / 4$. When the proportional gain is chosen, and based on $\zeta_{k}=1$ for $I_{\text {eff } m \text { max }}$, the derivative gain follows from:

$$
K_{\mathrm{DPk}}=\left(2 \sqrt{I_{\text {effk } \max }(\mathbf{q}) K_{\mathrm{PP} k}}\right),
$$

and will have the maximum value for $K_{\mathrm{PP} k}=K_{\mathrm{PP} k \max }$. The same analysis stands for the PI speed feedback controller in Eq. (15), where $K_{\mathrm{IS}}$ corresponds to $K_{\mathrm{PP}}$ in Eq. (16), and $K_{\mathrm{PS}}$ is obtained in the same way as $K_{\mathrm{DP}}$ in Eq. (17).

When the gains of the PD position/PI speed controller are chosen based on the structural resonance features, as described previously, to achieve a realistic simulation, the actuator capabilities, i.e., the maximum actuator torques, should be taken into account. Here, a model is adopted in which the maximum torque/ 
torque increment generated by an actuator based on the output of the feedback controller corresponds to the assumed maximum (allowed) controlled variable error. The corresponding gain should be applied to the output of the feedback controller within the simulation model.

Determination of the gain value that will provide a realistic simulation is based on the following model. The difference equation describing the actuator torque generated from the output of the PD position controller is [27]:

$$
K_{\mathrm{PP}} \Delta q(i)+K_{\mathrm{DP}}(\Delta q(i)-\Delta q(i-1))=\frac{\tau_{\mathrm{M}}(i)}{K_{\mathrm{TORP}}},
$$

where $\Delta q(i)$ is the positional error at sampling instant $i$ and $\tau_{\mathrm{M}}(i)$ is the torque generated by the actuator.

Likewise, in the case of a PI speed controller, the difference equation representing the torque increment $\Delta \tau_{\mathrm{M}}(i)$ generated by the actuator as a function of the speed error at sampling instant $i$ is [27]:

$$
K_{\mathrm{IS}} \Delta \dot{q}(i)+K_{\mathrm{PS}}(\Delta \dot{q}(i)-\Delta \dot{q}(i-1))=\frac{\Delta \tau_{\mathrm{M}}(i)}{K_{\mathrm{TORS}}}
$$

where $\Delta \dot{q}(i)$ is the speed error at the $i^{\text {th }}$ sampling instant.

Scaling factors $K_{\mathrm{TORP}}$ and $K_{\mathrm{TORS}}$, calculated from Eq. (18) and Eq. (19) for the maximum torque/torque increment, assumed maximum allowed controlled variable error, and feedback gains defined by Eqs. (16) and (17), provide values of the amplifier gains applied to the output of the feedback controllers in the presented simulation model. The maximum allowed position and speed errors should be chosen such that they are within the range of expected errors, in order to obtain gains $K_{\mathrm{TORP}}$ and $K_{\mathrm{TORS}}$ that would provide a realistic simulation to be obtained.

\section{RESULTS AND DISCUSSION}

\subsection{Simulation of Dynamics Effects of Interconnected CMS Links' Motions}

A method for the assessment of the mutual dynamic effects of the interconnected links' motions, given in Section 1.1, is applied for the CMS. In Fig. 5, joint actuating torque for the first axis $\tau_{1}$ obtained by the ID, Eq. (2), for the joint trajectories given in Fig. 3, is compared with the joint actuating torque $\tau_{1 Z}$ obtained by the ID, Eq. (2), for the same trajectory of axis 1, but with the trajectories of the other joints equalling zero $\quad\left(q_{2}=q_{3}=0, \quad \dot{q}_{2}=\dot{q}_{3}=0, \quad \ddot{q}_{2}=\ddot{q}_{3}=0\right) . \quad$ The difference is practically negligible. This indicates the insignificant influence of the motions of links 2 and 3 to the CMS arm motion, which is even more reduced due to the use of a gear reduction between the actuator and the link 1, as shown in Eq. (10). In the same way, simulations showed a large influence of the motion of the first axis on the actuating torques of links 2 and 3 owing to the large centrifugal and tangential acceleration forces produced by the CMS arm. In addition, significant mutual influence of the motions of links 2 and 3 was determined through their mutual contribution within actuating torques $\tau_{2}$ and $\tau_{3}$. Consequently, the following implementation of the control system is proposed: The first axis is controlled using feedback only, whereas a centralized control strategy is considered for the second and third axes. The latter is described later in this paper.

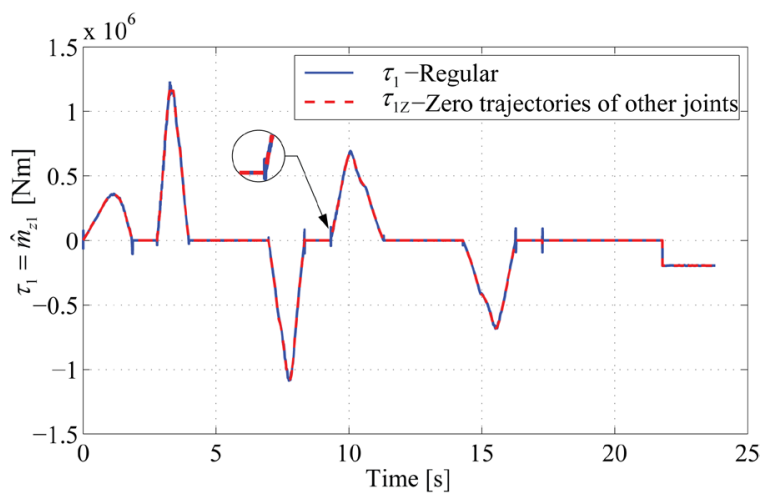

Fig. 5. The contribution of motions of second and third axes to the value of torque $\tau_{1}$

\subsection{Choice of actuators based on alD algorithm simulations}

The approximate ID algorithm described in Section 1.2 was tested for the chosen actuators. Massive simulations showed that modifications of the joint trajectories do not significantly influence the endeffector trajectories for a given choice of actuators with $\tau_{2,3 \max }=2 \cdot M_{2,3 \max }=21800 \mathrm{Nm}$. In Fig. 6 , the commanded acceleration at the centre of the gondola given by one of the most demanding application programs, and the same acceleration obtained as the output of the aID with the chosen actuators, are both given. Technical data and characteristics given in the manufacturer's configuration manual [24] indicate that the end-effector trajectory (absolute acceleration in the gondola centre) obtained after applying aID will significantly differ from the desired trajectory if a successively smaller motor is used. In this way, an actuator with the smallest weight and power with the potential to achieve the required joint motions is obtained. 


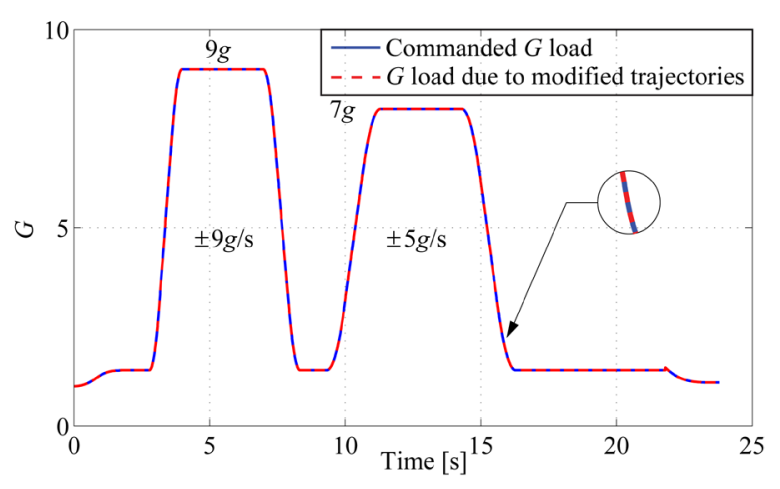

Fig. 6. Desired and modified absolute acceleration profiles

\subsection{Comparison of Motion Controller Performances for Axes 2 and 3}

A dynamic model-based control method that comprises feedback and feedforward computed torque compensation is considered for axes 2 and 3 in this study. Several Simulink models were designed to simulate and compare the controller performances for axes 2 and 3 achieved by position feedback, velocity feedback, and computed torque (CT) method with the same feedback controllers added. The reference position or speed in the Simulink models is given as a series of discrete values obtained from the trajectory planner given in Fig. 3 after an aID-based check of the trajectories is applied. The interpolation period $\Delta t$ is 5 $\mathrm{ms}$. The effective inertia is calculated for every interpolation period for links 2 and 3, as given in Eq. (11). Coefficients $d_{22}$ and $d_{33}$ are calculated using ID, Eq. (2), as the sum of the acceleration coefficients of $\ddot{q}_{2}$ and $\ddot{q}_{3}$, respectively. The moment of inertia of the motors is $I_{\mathrm{m} 2}=I_{\mathrm{m} 3}=1092 \cdot 10^{-2} \mathrm{kgm}^{2}$. For the trajectories given in Fig. $3, I_{\mathrm{eff} 2 \max }=2221.3 \mathrm{kgm}^{2}$ is obtained, relative to which the effective inertia varies up to $6.37 \%$, and $I_{\text {eff } 3 \max }=508.75 \mathrm{kgm}^{2}$ is obtained with the same variations of up to $15.47 \%$.

From CATIA, $\omega_{\mathrm{r} 2}=40.843 \mathrm{~Hz}$ is obtained as the lowest structural natural frequency of the CMS ring, whereas $\omega_{\mathrm{r} 3}=13.851 \mathrm{~Hz}$ is the lowest natural frequency of the gondola.

Linear time-invariant (LTI) models of the process given in Eq. (12) with a maximal value of the effective inertia, Eq. (11), for the trajectories given in
Fig. 3 are adopted. Gains $K_{\mathrm{PP} k} / K_{\mathrm{IS} k}$ and $K_{\mathrm{DP} k} / K_{\mathrm{PS} k}$ are chosen as the maximum gains given by Eqs. (16) and (17), respectively. Gains $K_{\mathrm{TORP} k}$ and $K_{\mathrm{TORS} k}$ obtained from Eqs. (18) and (19) are applied at the outputs of the feedback controllers. In Table 1, the process and feedback controller parameters for joints $k=2,3$ are given.

In models with feedback only, a disturbance is simulated as $\tau_{\mathrm{L} k}=\tau_{\mathrm{LD} k}, k=2,3$, obtained from ID, Eq. (10). In CT plus feedback control models, the load torques $\tau_{\mathrm{LD} k}, k=2,3$, calculated from, Eq. (10), are compensated for during every interpolation period, whereas the total load torque $\tau_{\mathrm{L} k}, k=2,3$ is simulated as $\tau_{\mathrm{L} k}=\tau_{\mathrm{LD} k}\left(1+A \sin \omega_{\mathrm{D}} t\right) k=2,3$. The term $\tau_{\mathrm{LD} k} A \sin \omega_{\mathrm{D}} t$ simulates the stochastic disturbances and uncertainties (errors in the estimates of the load torque, $\tau_{\mathrm{LD} k}$ ). Here, $t$ indicates the time, whereas the values $A$ and $\omega_{\mathrm{D}}$ are the amplitude and frequency of the disturbances. It should be noted that in the applied simulation models the torque limitation based on the model given in Eqs. (18) and (19) is included, the saturation of the torque generated from the output of the feedback controller is applied, as well as the saturation of the sum of the torques from the feedback and feedforward signals. Herein, the CT load torque compensation is effective only when the torque generated by the actuator due to feedback controller does not exceed the maximum actuator torque.

In Fig. 7, the position tracking of joints 2 and 3 is given as follows: a) joint 2, PD feedback controller, b) joint 3, PI feedback controller, c) joint 2, PD feedback controller together with the feedforward computed torque method, and d) joint 3, PI feedback controller together with feedforward computed torque method. Tracking errors are also given in Fig. 7 in e) for joint 2 , and in f) for joint 3 . In these simulations, $A=0.05$ (which suggests that the load torque estimation error is about $5 \%$ ), and $\omega_{\mathrm{D}}=100$.

The addition of feedforward cancelation of the nonlinear coupling terms in the dynamic model, Eq. (2), to the feedback controller achieves a considerable improvement in position tracking for joint 2. For joint 3 , improvement in position tracking is noticeable, but insignificant, considering the size of the tracking error. To decrease the error in position for link 3, a bigger torque actuator should be used. With the achieved

Table 1. Process and controller parameters for joints $k=2,3$ for the trajectories given in Fig. 3

\begin{tabular}{cccccccccc}
\hline Joint & $I_{\text {eff } k \text { max }}\left[\mathrm{kgm}^{2}\right]$ & $K_{\mathrm{PP} k}$ & $K_{\mathrm{DP} k}$ & $K_{\mathrm{IS} k}$ & $K_{\mathrm{PS} k}$ & $K_{\mathrm{TORP} k}$ & $K_{\mathrm{TORS} k}$ & $\Delta q_{k \max }[\mathrm{rad}]$ & $\Delta \dot{q}_{k \max }[\mathrm{rad} / \mathrm{s}]$ \\
\hline$k=2$ & 2221.3 & $3.65 \times 10^{7}$ & $5.7 \times 10^{5}$ & $3.65 \times 10^{7}$ & $5.7 \times 10^{5}$ & 0.115 & 0.115 & $5 \times 10^{-3}$ & $5 \times 10^{-3}$ \\
\hline$k=3$ & 508.75 & $9.63 \times 10^{5}$ & $4.43 \times 10^{4}$ & $9.63 \times 10^{5}$ & $4.43 \times 10^{4}$ & 4.14 & 4.14 & $5 \times 10^{-3}$ & $5 \times 10^{-3}$ \\
\hline
\end{tabular}



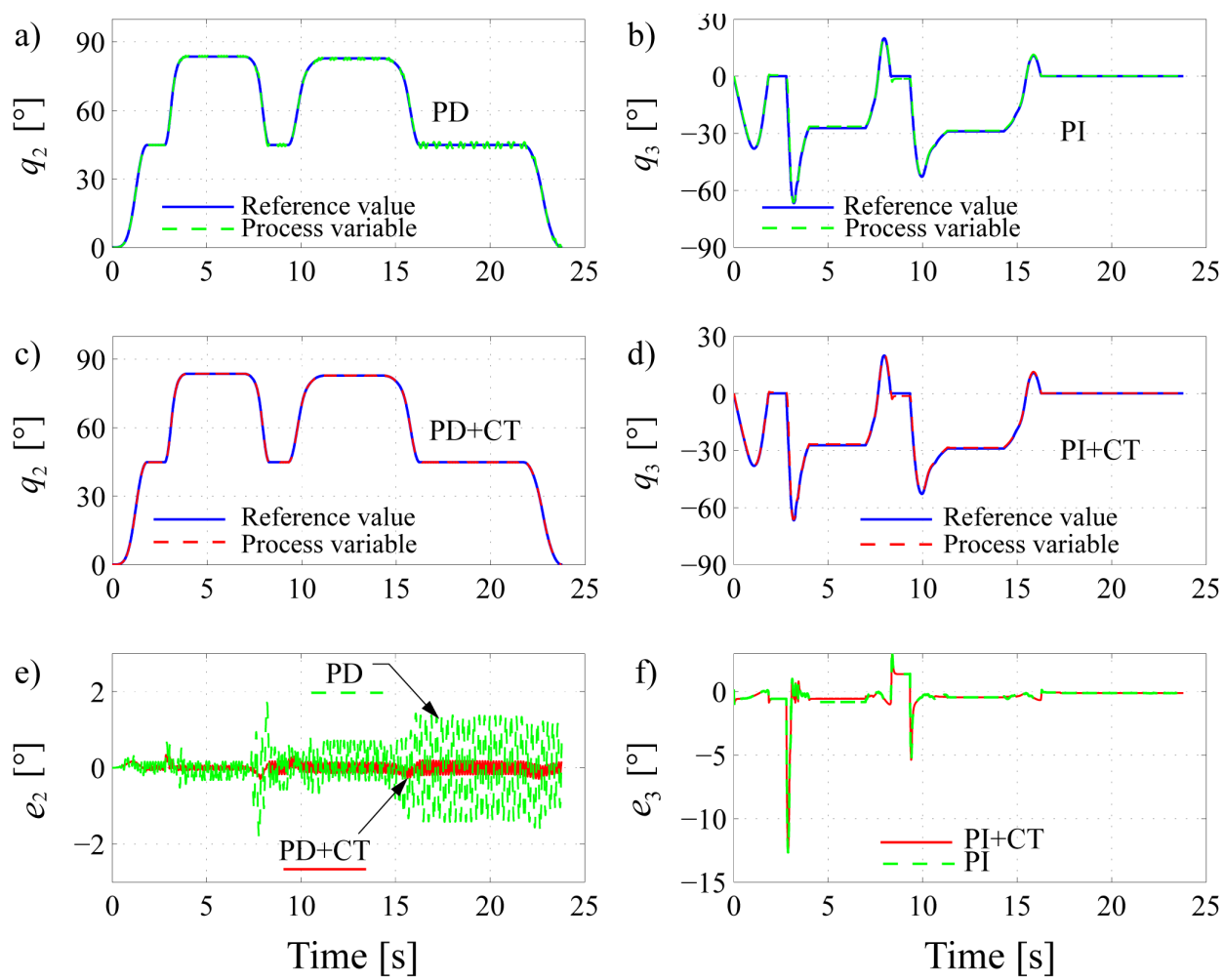

Fig. 7. Position tracking of a) joint 2, PD feedback controller (dashed/green); b) joint 3, Pl feedback controller (dashed/green); c) joint 2, PD feedback plus CT (dashed/red); d) joint 3, PI feedback plus CT (dashed/red); e) joint 2, position error with PD feedback (dashed/green) and PD feedback controller plus CT (solid/red); f) joint 3, position error with PI feedback controller (dashed/green) and PI feedback controller plus CT (solid/red)

tracking, the maximum absolute error in the absolute acceleration at the centre of the gondola is $0.47 \mathrm{~g}$.

\section{CONCLUSIONS}

The analysis described in this paper highlights the benefits of a dynamic model-based control system design for a CMS, which is a manipulator characterized by highly challenging motions. Based on appropriate inverse dynamics model simulations, a suitable choice of the control system and actuators, considering a satisfactory performance as well as the complexity and cost of the overall system, can be achieved. The use of an algorithm based on the approximate inverse dynamics model enables a compromise to be made between the power and weight of the chosen actuators. Realistic simulations of the controlled process for the ring and gondola axes, which account for structural natural frequencies, actuator torque limits, and inertia reflected on the rotor shafts, were conducted, enabling a comparison between the standard decentralized feedback control and a dynamic model-based control strategy consisting of a computed torque method with the addition of feedback. Simulations demonstrated the significant benefits in position tracking with disturbance cancelation achieved using a computed torque method added to the feedback when an actuator that is able to achieve the required motion is applied. Future work will be focused on implementing the method presented in this paper to other systems. Furthermore, alternative centralized control methods will be considered.

\section{ACKNOWLEDGEMENTS}

This research has been supported by a research grant of the Serbian Ministry of Education, Science and Technological Development under numbers TR 35023 and TR 35006.

\section{REFERENCES}

[1] Kvrgic, V.M., Vidakovic, J.Z., Lutovac, M.M., Ferenc, G.Z., Cvijanovic, V.B. (2014). A control algorithm for a centrifuge motion simulator. Robotics and Computer-Integrated 
Manufacturing, vol. 30, no. 4, p. 399-412, D0l:10.1016/j. rcim.2014.01.002.

[2] Albery, W.B. (2004). Acceleration in other axes affects $+G z$ tolerance: dynamic centrifuge simulation of agile flight. Aviation, Space, and Environmental Medicine, vol. 75, no. 1, p. 1-6.

[3] Hartenberg, R.S., Denavit, J. (1964). Kinematic Synthesis of Linkages. McGraw-Hill, New York.

[4] Slotine, J.-J.E., Li, W. (1987). On the adaptive control of robot manipulators. The International Journal of Robotics Research, vol. 6, no. 3, p. 49-59, Dol:10.1177/027836498700600303.

[5] Wang, H. (2017). Adaptive control of robot manipulators with uncertain kinematics and dynamics. IEEE Transactions on Automatic Control, vol. 62, no. 2, p. 948-954, D0l:10.1109/ TAC.2016.2575827.

[6] Ferrara, A., Incremona, G.P. (2015). Design of an integral suboptimal second-order sliding mode controller for the robust motion control of robot manipulators. IEEE Transactions on Control Systems Technology, vol. 23, no. 6, p. 2316-2325, DOI:10.1109/TCST.2015.2420624.

[7] Chen, C.-S. (2008). Dynamic structure neural-fuzzy networks for robust adaptive control of robot manipulators. IEEE Transactions on Industrial Electronics, vol. 55, no. 9, p. 34023414, DOI:10.1109/TIE.2008.926778.

[8] Jerbic, B., Nikolic, G., Chudy, D., Svaco, M., Sekoranja, B. (2015). Robotic application in neurosurgery using intelligent visual and haptic interaction. International Journal of Simulation Modelling, vol. 14, no. 1, p. 71-84, D0l:10.2507/ IJSIMM14(1)7.290.

[9] Li, X., Cheah, C.C. (2014). Adaptive neural network control of robot based on a unified objective bound. IEEE Transactions on Control Systems Technology, vol. 22, no. 3, p. 1032-1043, DOI:10.1109/TCST.2013.2293498.

[10] Erkaya, S. (2018). Effects of joint clearance on motion accuracy of robotic manipulators. Strojniški vestnik Journal of Mechanical Engineering, vol. 64, no. 2, p. 82-94, D0I:10.5545/sv-jme.2017.4534.

[11] Klobučar, R., Čas, J., Šafarič, R., Brezočnik, M. (2008). Uncalibrated visual servo control with neural network. Strojniški vestnik - Journal of Mechanical Engineering, vol. 54, no. 9, p. 619-627.

[12] Peng, J., Liu, Y., Wang, J. (2014). Fuzzy adaptive output feedback control for robotic systems based on fuzzy adaptive observer. Nonlinear Dynamics, vol. 78, no. 2, p. 789-801, DOI:10.1007/s11071-014-1477-z.

[13] Das, M.T., Dulger, L.C., Das, G.S. (2013). Robotic applications with particle swarm optimization (PSO). IEEE International Conference on Control, Decision and Information Technologies, p. 160-165, Dol:10.1109/CoDIT.2013.6689537.

[14] Hrelja, M., Klancnik, S., Irgolic, T., Paulic, M., Jurkovic, Z., Balic, J., Brezocnik, M. (2014). Particle swarm optimization approach for modelling a turning process. Advances in Production Engineering \& Management, vol. 9, no. 1, p. 2130, DOI:10.14743/apem2014.1.173.

[15] Mei, J., Zang, J., Ding, Y., Xie, S., Zhang, X. (2017). Rapid and automatic zero-offset calibration of a 2-DOF parallel robot based on a new measuring mechanism. Strojniški vestnik
- Journal of Mechanical Engineering, vol. 63, no. 12, p. 715724, DOl:10.5545/sv-jme.2017.4529.

[16] Chung, W.K., Fu, L.-C., Hsu, S.-H. (2008). Motion control. Siciliano, B., Khatib, 0. (eds.). Springer Handbook of Robotics. Springer-Verlag, Berlin Heidelberg, p. 133-160, Dol:10.1007/978-3-540-30301-5_7.

[17] Sciavicco, L., Siciliano, B., Villani, L., Oriolo, G. (2012). Robotics-Modelling, Planning and Control. Advanced Textbooks in Control and Signal Processing Series. SpringerVerlag London, London, D0I:10.1007/978-1-84628-642-1.

[18] Schumann, J., Gupta, P., Liu, Y. (2010). Application of neural networks in high assurance systems: A survey. Schumann, J., Liu, Y. (eds). Applications of Neural Networks in High Assurance Systems. Studies in Computational Intelligence, vol. 268, Springer-Verlag, Berlin Heidelberg, Dol:10.1007/9783-642-10690-3_1.

[19] Tsai, M.-H., Shih, M.-C. (2009). G-load tracking control of a centrifuge driven by servo hydraulic systems. Proceedings of the Institution of Mechanical Engineers, Part G: Journal of Aerospace Engineering, vol. 223, no. 6, p. 669-682, DOI:10.1243/09544100JAER0519.

[20] Chen, Y.C., Repperger, D.W. (1996). A study of the kinematics, dynamics and control algorithms for a centrifuge motion simulator. Mechatronics, vol. 6, no. 7, p. 829-852, DOl:10.1016/0957-4158(96)00018-9.

[21] Markiewicz, B.R. (1973). Analysis of the Computed Torque Drive Method and Comparison with Conventional Position Servo for a Computer-Controlled Manipulator (NASA Technical Memorandum 33-601), California Institute of Technology, Pasadena.

[22] Lutovac, M., Ferenc, G., Kvrgić, V., Vidaković, J., Dimić, Z. (2012). Robot programming system based on L-IRL programming language. Acta Technica Corviniensis-Bulletin of Engineering, vol. 5, no. 2, p. 27.

[23] Vidakovic, J., Lazarevic, M., Kvrgic, V., Dančuo Z., Lutovac, M. (2013). Comparison of numerical simulation models for open loop flight simulations in the human centrifuge. PAMM, vol. 13, no. 1, p. 485-486, D0l:10.1002/pamm.201310235.

[24] SINAMICS Configuration Manual: 1FW6 Built-In Torque Motors (2009). Siemens Support, Erlangen.

[25] Craig, J.J. (2005). Introduction to Robotics: Mechanics and Control (3rd ed.). Pearson Prentice Hall, Upper Saddle River.

[26] Scheinman, V., McCarthy, J.M. (2008). Mechanisms and actuation. Siciliano, B., Khatib, O. (eds.). Springer Handbook of Robotics. Springer-Verlag, Berlin Heidelberg, p. 67-86, DOI:10.1007/978-3-540-30301-5_4.

[27] Vukosavic, S. (2007). Digital Control of Electrical Drives. Springer-Verlag US, New York, DOI:10.1007/978-0-387-485980.

[28] Spong, M.W., Hutchinson, S., Vidyasagar, M. (2006). Robot dynamics and control. John Wiley \& Sons, Hoboken.

[29] Lee, H.S., Tomizuka, M. (1996). Robust motion controller design for high-accuracy positioning systems. IEEE Transaction on Industrial Electronnics, vol. 43, no. 1, p. 48-55, DOI:10.1109/41.481407.

[30] Aström, K., Hägglund, T. (1995). PID Controllers: Theory, Design, and Tuning, (2nd ed.) Isa, Research Triangle Park NC. 